Revue internationale P.M.E.

Économie et gestion de la petite et moyenne entreprise

\title{
Les mesures de perception de la qualité totale dans les PMI : résultats de recherche
}

\section{Manouchehr Ansari}

Volume 12, numéro 4, 1999

URI : https://id.erudit.org/iderudit/1008663ar

DOI : https://doi.org/10.7202/1008663ar

Aller au sommaire du numéro

Éditeur(s)

Presses de l’Université du Québec

ISSN

0776-5436 (imprimé)

1918-9699 (numérique)

Découvrir la revue

Citer cet article

Ansari, M. (1999). Les mesures de perception de la qualité totale dans les PMI : résultats de recherche. Revue internationale P.M.E., 12(4), 9-31.

https://doi.org/10.7202/1008663ar
Résumé de l'article

L'ambition de ce travail est d'explorer la problématique de révolution du concept de la qualité totale dans les PMI sous-traitantes des secteurs de l'automobile et de l'électrique-électronique de Haute et de Basse-Normandie. Ce sont, en effet, les acteurs de ces secteurs d'activité qui nous ont servi pour illustrer nos propos afin d'évaluer les résultats de notre étude qualitative. Elle expose i'ensemble des données générales de l'enquête et de la méthodologie utilisée. Celle-ci insiste sur le choix des secteurs retenus, sur la construction du questionnaire et du modèle conçus à partir du Prix européen de la qualité ainsi que de l'analyse des résultats obtenus et leur interprétation. 


\title{
Les mesures de perception de la qualité totale dans les PMI : résultats de recherche
}

\author{
Manouchehr ANSARI \\ Institut d'administration des entreprises de Rouen
}

\section{MOTS CLÉS}

\section{Qualité - Gestion qualité - Management - Normes ISO 9000 Certification - Évaluation - PMI sous-traitantes de l'automobile et électrique-électronique}

\begin{abstract}
RÉSUMÉ
L'ambition de ce travail est d'explorer la problématique de l'évolution du concept de la qualité totale dans les PMI sous-traitantes des secteurs de l'automobile et de l'électrique-électronique de Haute et de Basse-Normandie. Ce sont, en effet, les acteurs de ces secteurs d'activité qui nous ont servi pour illustrer nos propos afin d'évaluer les résultats de notre étude qualitative. Elle expose l'ensemble des données générales de l'enquête et de la méthodologie utilisée. Celle-ci insiste sur le choix des secteurs retenus, sur la construction du questionnaire et du modèle conçus à partir du Prix européen de la qualité ainsi que de l'analyse des résultats obtenus et leur interprétation.
\end{abstract}

\section{L'AUTEUR}

MANOUCHEHR ANSARI est docteur en sciences de gestion. Ses travaux de recherche portent sur le TQM et la théorie des organisations, dans le cadre du CREGO (Centre de recherche et d'étude en gestion des organisations) à l'Institut d'administration des entreprises de Rouen. Adresse: 43, rue Pierre-Girard, 14000 Caen, France.

Je tiens à remercier les lecteurs anonymes pour la pertinence de leurs remarques et de leurs suggestions.

Je remercie aussi monsieur le professeur Éric Vatteville, directeur de l'IAE de Rouen, de m'avoir apporté son aide et son soutien constant ; sans lui, cet article n'aurait pu être réalisé. 


\begin{abstract}
This study explores the evolution of the TQM (total quality management) concept in subcontracting SMEs (small and medium-sized enterprises) of the automotive and electric/electronic sectors in Upper and Lower Normandy. These actors were used to depict and evaluate the results of our qualitative study. The paper presents the general data from the study as well as the methodology, insisting on the selection of the sectors and the development of the questionnaire and model, which were inspired from the "Prix européen de la qualité". The analysis and interpretation of the results are also covered.
\end{abstract}

\title{
RESUMEN
}

La ambición de este trabajo es explorar la problemática de la evolución del concepto de calidad total en las Pequeñas y Medianas Industrias (PyMI) subcontratantes de los sectores de actividad del automóvil y la electricidad-electrónica en Alta y Baja Normandia. Estas empresas que son, efectivamente, actores de estos sectores de actividad, han servido para ilustrar nuestros propósitos, con el fin de evaluar los resultados de nuestro análisis cualitativo. Expone la totalidad de las informaciones generales de la investigación y la metodología utilizada. Esta úitima insiste sobre la selección de los sectores escogidos, la construcción del cuestionario y el modelo concebido a partir del Premio europeo de la calidad, así como el análisis de los resultados obtenidos y sus interpretaciones.

\section{ZUSAMMENFASSUNG}

Das Ziel dieser Arbeit ist die Problematik der Entwicklung eines Total-QualitySystems im Bereich der KMU Zwischenhändler im Automobil- sowie im Elektrosektor der "Haute- und Basse-Normandie" zu erforschen. Die Akteure dieser Sektoren dienten als Basis, eine qualitative Studie zu illustrieren. Die Studie veranschaulicht den Ablauf und die angewandte Methodik. Die Wahl der ausgewählten Sektoren und der Aufbau des Fragebogens basieren auf dem Modell des Europäischen Qualitätspreises und dessen Interpretation.

\section{Introduction}

La mutation enregistrée ces dernières années dans les PMI a nécessité une coordination efficace des activités des unités individuelles qui composent la qualité totale. Cette nécessité s'explique par la pression de la concurrence qui oblige actuellement toutes les entreprises à définir globalement leur domaine, à diminuer les délais d'arrivée de leurs produits sur le marché et, enfin, à redoubler d'effort pour manager le risque, le service, la qualité et les coûts à un niveau mondial. 
La pression de la concurrence internationale les met au défi d'augmenter leur performance définie traditionnellement à travers la part de marché, le retour sur investissement, la satisfaction du client, l'évaluation de la qualité et de réexaminer aussi la façon de mesurer ces performances. Pour développer ce comportement, il faut élargir le concept de la qualité totale dans la gestion des PMI. Durant les années 1980, parmi les dispositifs concrets au service de ces démarches, les cercles de qualité ont été les premiers à occuper une place dominante. Aujourd'hui, le mouvement de la qualité et les normes ISO 9000 tentent de répondre à l'ensemble de ces exigences par l'application de nouvelles normes de travail. La survie de l'entreprise apparaît alors liée à un ensemble de transformations tant au niveau des structures de l'organisation qu'à celui des pratiques managériales dans la recherche d'une optimisation constante de la production. La mise en place d'actions qualité, dans ce processus de l'innovation, passe par de nouvelles techniques, de nouvelles méthodes de gestion. Elle s'accompagne d'une redéfinition des responsabilités et des compétences au travail, voire d'une transformation de la manière de faire et de penser de l'ensemble des membres de l'organisation (Bonnet, 1994).

Dans cette perspective, il serait intéressant de faire une réflexion sur les modèles implicites de la gestion des PMI, qui véhiculent les démarches de normalisation (ISO 9000 - ISO 14000) et le management de la qualité totale, en particulier les trophées des différents prix de la qualité. En outre, plusieurs raisons expliquent la décision d'intégrer ces deux concepts aux valeurs des entreprises (Igalent, 1992). À cette fin, l'intérêt de cette recherche est d'apporter aux dirigeants des PMI des informations sur l'évolution et la nécessité de l'application d'une démarche de qualité totale. La démarche qualité par le biais de la certification ISO 9000 a donc des effets appréciables en ce qui concerne les opérations administratives des PMI : une procédure de paie, un suivi administratif de la formation ou la production d'informations sociales au sein de laquelle elle influence les choix d'organisation, ainsi que la totalité du système de gestion et des procédures, les attitudes et les comportements des hommes et des femmes au travail. C'est à partir de ces éléments que nous essaierons de présenter le résultat de notre enquête, en mettant l'accent sur la gestion de la qualité totale dans les PMI. Mais avant de présenter les principaux résultats de notre étude, il convient de préciser le cadre dans lequel elle a été menée.

\section{Cadre de la recherche}

Plusieurs objectifs ont été assignés à cette recherche. Ces objectifs correspondent à la formulation de notre exposé et nous permettent d'appréhender le processus de mise en œuvre de la démarche qualité et son développement dans les PMI (figure 1). 
FIGURE 1

Exposé du cheminement de notre recherche

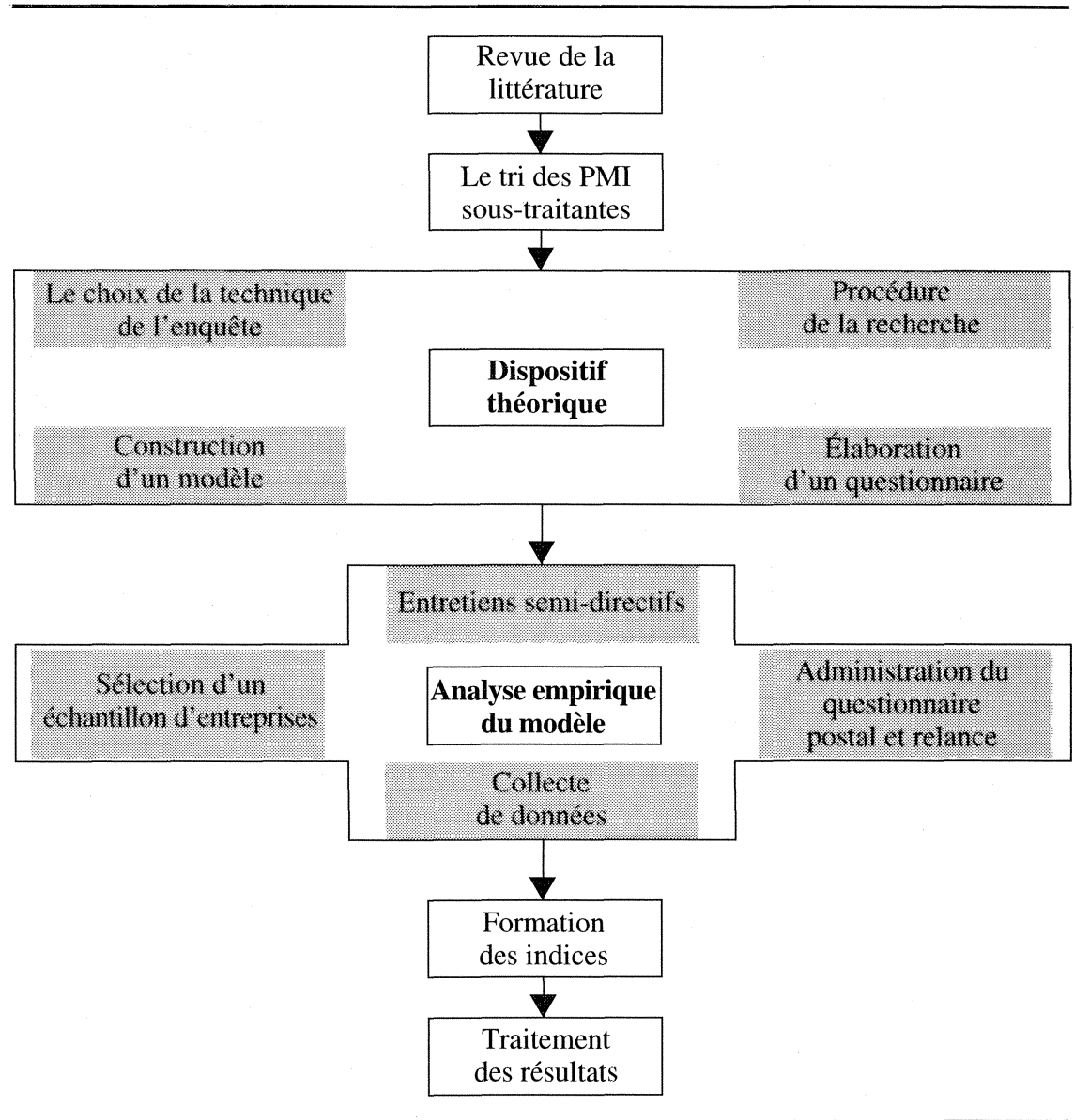

Les aspects développés ci-dessus ont été menés en deux parties. Dans la première, nous avons établi un état de lieu en procédant à la revue de la littérature sur le concept de qualité. Il nous faut en effet partir de l'existant pour comprendre pour quelle raison le concept est proliférant, décrire les valeurs de diffusion de la thématique qualité et analyser l'évolution de son contexte pour les entreprises. Dans la seconde, nous avons présenté les tendances actuelles des pratiques de la qualité totale dans les PMI sous-traitantes de l'automobile et de l'électrique-électronique, à travers une étude empirique. Cette dernière s'appuie sur des entretiens semidirectifs et insiste sur le choix des secteurs retenus, sur le choix du terrain d'observation, sur la construction du questionnaire et du modèle conçus à partir du Prix 
européen de la qualité. Tous ces éléments nous ont permis d'identifier les objectifs actuellement poursuivis en matière de démarche qualité et de normes ISO 9000. Ceci nous amène à poser la problématique suivante.

\section{Problématique}

La problématique a donc consisté à mettre en évidence l'impact des attributs du concept de qualité dans les PMI et à relever dans quelle mesure et dans quelles circonstances les responsables des firmes l'utilisent dans leur travail de chef d'entreprise. Car ce sont les acteurs de ces entreprises qui nous ont servi, en effet, pour illustrer notre recherche, l'objectif étant de réaliser un test pour évaluer la démarche qualité. Ainsi, c'est à partir de ces éléments qu'une méthode de recueil et d'analyse des données a été élaborée, et ce, afin de mieux connaître les conditions, les difficultés et les limites d'une évolution de la démarche qualité allant dans le sens d'une amélioration continue, au sein des PMI régionales. Deux questions de recherche ont constitué le fondement de ce travail.

\section{Les principes fondamentaux de la recherche}

Premièrement, en quoi la gestion de la qualité permet-elle de mettre en œuvre les options nouvelles et quelles en sont les conséquences sur l'organisme de l'entreprise ?

Deuxièmement, comment la stratégie et les objectifs qualité sont-ils diffusés par les dirigeants au sein des PMI ? Sont-ils déclinés en plans d'action ? Les résultats de cette action sont-ils évalués?

Des éléments de réponse à ces questions permettraient aux responsables des entreprises d'approfondir leur connaissance des modes d'organisation possibles et de mieux appréhender les processus d'évolution du concept qualité mis en place. Or, nous avons validé empiriquement ces éléments théoriques auprès des PMI. Pourquoi ce choix des PMI?

Pour la simple raison qu'elles prennent une place non négligeable dans le tissu industriel des trois grandes puissances industrielles que sont l'Union européenne, les États-Unis et le Japon. Le poids des PMI est cependant différent d'un pays à l'autre. Il est lié à l'histoire économique et industrielle de chacun des pays qui s'opposent sur ce terrain, de l'Allemagne dont l'industrie est très concentrée, au Japon, « pays de la PMI » à proprement parler. La forte présence de PMI ne semble donc pas, au vu des résultats industriels japonais, être un handicap au dynamisme économique d'un pays. Celui-ci dépend néanmoins des liens qu'entretiennent entre elles les entreprises de grande taille et celles de dimension plus modeste ${ }^{1}$. Malgré l'absence d'harmonisation dans la définition des PMI qui rend difficile leurs

1. Ministère de l'Industrie (1992), Les PMI dans le contexte international, Paris. 
comparaisons, elles bénéficient d'un grand prestige, aussi bien dans le secteur automobile qu'électrique-électronique. Ces entreprises conçoivent et réalisent depuis toujours des pièces et des appareils complets, seules ou en coopération avec d'autres grandes entreprises nationales ou européennes (Esposito et Raffa, 1992). Or, elles jouent un rôle essentiel dans le développement des entreprises et l'insertion de l'innovation dans le tissu industriel. Leur flexibilité, leur adaptabilité ou leur créativité sont des atouts qui font défaut à la grande entreprise. Plusieurs enquêtes vont dans ce sens, en montrant que les PMI sont plus résistantes et plus performantes que les grandes entreprises dans un contexte de crise. Par ailleurs, on sait qu'aujourd'hui les grandes entreprises sont peu créatrices d'emplois et qu'en revanche les PMI se montrent beaucoup plus dynamiques dans ce domaine (Auregan, 1996). Aussi, le choix de ces secteurs nous paraît-il significatif pour plusieurs raisons.

\section{Caractéristiques des secteurs retenus}

Premièrement. Les secteurs de l'automobile et de l'électrique-électronique sont représentatifs des industries qui utilisent un ensemble de technologies élaborées pour d'autres applications.

Deuxièmement. Les entreprises de ces secteurs opèrent sans discontinuité avec des normes de qualité et de fiabilité.

Troisièmement. Depuis toujours, les rapports entre donneurs d'ordres et sous-traitants y sont réglés dans le but d'assurer des normes de qualité élevées au produit.

Dans cette perspective, la souplesse des structures de la PMI, sa rapidité dans la prise des décisions, le potentiel d'innovation dont elle dispose et sa préférence stratégique pour une démarche de la qualité totale peuvent constituer des conditions favorables à la performance de cette catégorie d'entreprise. À partir de la problématique et des faits observés, nous avons pu bâtir une méthodologie de recherche.

\subsection{La méthodologie de la recherche}

Tout d'abord, une enquête a été réalisée auprès de $10 \mathrm{PMI}^{2}$ certifiées ou en cours de certification, laquelle nous a permis de dégager quelques hypothèses dans le but d'élaborer un modèle. Le modèle que nous avons constitué s'inspire plus particu-

2. Nous pouvons justifier le choix de notre échantillon à partir des considérations suivantes : en premier lieu, on trouve dans ce secteur les entreprises qui progressent par la remise en cause de leur système d'organisation. Il suffit de consulter les différents numéros de certaines revues professionnelles et spécialisées (comme l'Usine nouvelle et Qualité en mouvement) pour se rendre compte que le secteur choisi est véritablement engagé dans le processus d'application des normes internationales. En second lieu, et les entretiens exploratoires nous l'ont confirmé, la démarche de la qualité totale y est une pratique assez largement utilisée. 
lièrement de la structure du modèle européen de management par la qualité totale qui prévaut actuellement en Europe. L'analyse de celui-ci nous a permis de dégager les composantes importantes du système du Prix européen de la qualité, susceptibles d'expliquer l'évaluation de la qualité des PMI et la perception des outils du concept de la qualité totale par le management, c'est-à-dire l'implication de la direction et la performance de ces outils dans l'application du processus. Ce type de modèle fournit une image ponctuelle de la situation de l'organisation. Cette image est généralement exprimée en termes de points forts, de domaines à améliorer et de scores. Rien ne changera dans l'organisation, après cette procédure, si des actions ne sont pas entreprises au niveau des résultats. Cette prise de conscience effectuée, l'entreprise pourra appliquer le référentiel de manière qualitative et pratiquer ainsi l'auto-évaluation de ses propres performances (EFQM, 1996). À cet effet, les objectifs assignés à cette recherche nous ont conduit à la formulation de neuf critères dans le modèle que nous avons élaboré (figure 2).

Figure 2

Modèle conceptuel de la qualité et critères correspondants

\begin{tabular}{|c|c|c|c|c|}
\hline $\begin{array}{l}\text { 7. Gestion des } \\
\text { documents } \\
90 \text { points } \\
(9 \%)\end{array}$ & $\begin{array}{l}\text { 1. Leadership } \\
100 \text { points } \\
(10 \%) \\
\text { 2. Politique et } \\
\text { stratégie } \\
80 \text { points } \\
(8 \%) \\
\text { 3. Gestion du } \\
\text { personnel } \\
90 \text { points } \\
(9 \%)\end{array}$ & $\begin{array}{l}\text { 8. Outils du } \\
\text { management } \\
\text { de la qualite } \\
\text { totale } \\
140 \text { points } \\
(14 \%)\end{array}$ & $\begin{array}{l}\text { 4. Processus } \\
140 \text { points } \\
(14 \%) \\
\text { 5. Satisfaction } \\
\text { du client } \\
200 \text { points } \\
(20 \%) \\
\text { 6. Satisfaction } \\
\text { du personnel } \\
90 \text { points } \\
(9 \%)\end{array}$ & $\begin{array}{l}\text { 9. Role des } \\
\text { pourvoirs } \\
\text { institution- } \\
\text { nels } \\
70 \text { points } \\
(7 \%)\end{array}$ \\
\hline
\end{tabular}

Note: Les pourcentages indiqués sont ceux utilisés pour l'attribution du Prix européen de la qualité. Source: Constitution à partir du modèle du Prix européen de la qualité.

Ces critères constituent un référentiel destiné à apprécier la manière dont les entreprises appliquent les principes de la qualité totale et les progrès accomplis par l'organisation sur la voie de l'excellence. Pour répondre aux objectifs fixés et aux critères définis dans le cadre de la recherche, nous avons procédé à l'analyse empirique de ce modèle.

\subsection{Analyse empirique du modèle}

À partir de celle-ci, nous avons procédé à une collecte de données, lesquelles ont été recueillies auprès des quelques professionnels dans le cadre d'un entretien exploratoire des informations concernant la démarche de qualité totale. Ces 
entretiens visaient deux objectifs : d'une part, comparer les variables théoriques identifiées à partir des questionnaires sur la qualité totale et, d'autre part, préciser certaines variables latentes. Ils ont donc été menés auprès de responsables de PMI ou de responsables qualité afin d'apporter une première validation de ce modèle en vérifiant que le choix des variables était légitime. C'est à partir de ces informations que le recueil de données fut réalisé de mars 1996 à janvier 1997. Pour constituer l'échantillon de l'enquête tout en tenant compte du secteur d'activité, les critères descriptifs suivants avaient été retenus :

- être d'une taille comprise entre 20 et 500 salariés. Pour plus de facilité, le choix s'est porté particulièrement sur les PMI qui se sont implantées en Haute et en Basse-Normandie ;

- être certifié ISO 9000 ou en cours de certification.

Nous avions retenu ces critères afin de nous assurer que l'organisation disposait d'un service qualité (application des normes ISO 9000) ou d'une démarche de qualité totale. Les résultats montrent que les entreprises de notre échantillon sont organisées de manière classique autour d'une approche de la qualité totale ; 41 questionnaires complétés sur 180 furent retournés, ce qui représente un taux de réponse de $23 \%$. Pour l'ensemble de l'échantillon, cela est donc acceptable et, par conséquent, la répartition des PMI et le nombre des réponses obtenues par département figure dans le tableau 1 , de la manière suivante.

\section{TABLEAU 1}

Répartition des PMI interrogées et nombre de réponses obtenues par département

\begin{tabular}{lcccccc}
\hline & $\begin{array}{c}\text { Nombre d'établissements } \\
\text { contactés }\end{array}$ & \multicolumn{4}{c}{$\begin{array}{c}\text { Nombre de réponses } \\
\text { obtenues }\end{array}$} \\
\hline Département & $\begin{array}{c}\text { Secteur } \\
\text { automobile }\end{array}$ & $\begin{array}{c}\text { Secteur électrique } \\
\text { et électronique }\end{array}$ & Automobile & Cumul & Électrique et \\
Électronique & Cumul \\
Seine-Maritime & 35 & 30 & 8 & 8 & 10 & 10 \\
Eure & 19 & 17 & 5 & 17 & 3 & 17 \\
Calvados & 12 & 7 & 3 & 20 & 1 & 18 \\
Manche & 12 & 6 & 2 & 22 & 1 & 19 \\
Orne & & & & & & \\
\hline Total & $\mathbf{1 0 0}$ & $\mathbf{8 0}$ & $\mathbf{2 2}$ & & & \\
\hline
\end{tabular}

\section{Résultats descriptifs de l'efficacité de la démarche qualité}

La qualité a toujours été présente dans les entreprises et leur a permis de connaître un développement rapide durant les 15 dernières années. Aujourd'hui, le développement de composants de plus en plus complexes dans un marché très concurrentiel 
nécessite une mutation profonde des méthodologies actuellement utilisées, touchant les éléments techniques mais également l'ensemble de l'environnement de la conception et de la fabrication des produits.

Pour répondre à cet important enjeu, les PMI ont uni leurs efforts de recherche avec les donneurs d'ordres et élaboré une nouvelle approche qualité commune (Convention performance de Haute et de Basse-Normandie) pour développer le partenariat, renouveler et élargir le prix régional de la qualité, afin de faire connaître, de la façon la plus large, les succès et les acquis des actions lancées dans la région. Le but est de faire progresser la qualité dans les entreprises régionales par une sensibilisation de tous les acteurs économiques. Les démarches qualité concernent en effet les entreprises de toute taille, de tout secteur d'activité, les sous-traitants comme les fournisseurs. Elles touchent en outre les entreprises de service, les services publics et l'administration, qui ont aujourd'hui à relever les nouveaux défis que représentent la satisfaction des usagers et la maîtrise des coûts. Donc, la mise en place d'une méthodologie de ce concept est exigée, aujourd'hui, par tous et devient par là même un modèle de référence pour les entreprises qui concourent au prix de la qualité. C'est dans cette optique que nous allons analyser comment les principes de la qualité totale sont utilisés pour l'étude des objectifs assignés à ce concept tant dans la littérature managériale que dans les entreprises.

\subsection{Valeurs assignées au concept qualité dans la littérature managériale}

Le concept qualité dans une entreprise a pour mission de garantir l'atteinte des objectifs fixés et de créer une dynamique d'amélioration conduisant à l'excellence. Des recherches sont en cours pour moderniser l'appareil méthodologique dans ce domaine. La gestion de la qualité apparaît alors comme un nouveau mode d'organisation de l'entreprise et peut s'inscrire en continuation des théories de l'organisation dominante. Elle vise à améliorer la performance globale des PMI et à suivre la procédure, à la respecter et à rechercher en permanence à l'améliorer. La littérature managériale sur la qualité propose à cet effet de nombreuses démarches et définitions (Gogue, 1987 ; Périgord, 1987 ; Peyraut, 1988 ; Saval et Zardet, 1989 ; Douchy, 1990 ; Moret, 1991 ; Jocou et Lucas, 1992 ; Ziane, 1993). Le développement du concept de la qualité totale est associé à d'autres personnalités importantes : Deming, Juran, Feigenbaum, Crosby et Ishikawa. Elle doit son développement aux apports d'experts en management comme Peter Drucker ou McGregor, mais aussi à celui de l'école japonaise. Ces démarches se sont institutionnalisées dans des instances ministérielles et dans des associations spécialisées (MFQ et AFAQ). Parallèlement ont émergé dans les chambres de commerce et d'industrie, des institutions chargées de promouvoir la qualité et de stimuler l'émergence d'entreprises certifiées (Neuville, 1996). 
Un examen de recherche dans le domaine montre que le concept de la qualité recouvre en fait un vaste éventail de sujets et d'approches. Gélinier (1993) décrit le concept de qualité comme un mode d'action qui tisse étroitement l'aspect technico-économique et l'aspect humain avec la formation et la dynamique de groupe, ce qui est puissamment motivant pour les hommes qui permettent au système de gestion de s'améliorer pour plus de compétitivité.

Martin (1992), Esposito et Raffa (1992) définissent la qualité comme une institution nouvelle qui incite les acteurs économiques à investir dans un processus de transformation majeure du fonctionnement interne et des rapports externes de leur entreprise. Pour les PMI traditionnellement engagées dans les sous-traitances industrielles, l'incitation devient un impératif de survie depuis que les grands donneurs d'ordres se dotent de politique d'assurance qualité qui changent les critères de sélection et d'évaluation du rendement des sous-traitants (Belley et Billette, 1995). Plus intéressant, le regard porté sur la qualité, appliqué à l'ensemble de l'entreprise, a amené les PMI à modifier leurs organisations. Elles ont ainsi apporté des transformations profondes à leurs méthodes de travail et à la conception de leur outil de production afin de les rendre plus flexibles, et ce, dans le but de favoriser l'adaptabilité aux changements qualitatifs de la demande (Bonnet, 1994).

Dans un tel contexte, le concept de la qualité apparaît comme un mécanisme susceptible de maintenir et d'améliorer la compétitivité des entreprises, qui peuvent ainsi soutenir la concurrence. Les PMI doivent se fixer des objectifs élevés en termes de qualité, de rapidité de développement de nouveaux produits, de leur mise sur le marché et de flexibilité. Cela exige une maîtrise des changements organisationnels ou technologiques et la capacité d'innover (Lefebvre et Bourgault, 1996). La survie de l'entreprise apparaît alors liée à un ensemble de transformations tant dans les structures de l'organisation que dans les pratiques managériales dans la recherche d'une optimisation constante de la production. Ces transformations accompagnent les différentes phases de la qualité, dépassent les changements de méthodes et de techniques (Bonnet, 1994).

\subsection{Valeurs assignées au concept de la qualité dans les PMI}

Une revue de la littérature managériale nous a permis d'élaborer un modèle (figure 2) expliquant le processus d'application d'une démarche de la qualité totale. Plusieurs variables explicatives ont ainsi été identifiées. À partir de celles-ci, nous avons attribué une note à chacune des parties des critères en combinant plusieurs variables selon les réponses données à nos questionnaires. La feuille de synthèse nous a permis de combiner les notes attribuées aux critères pour en recueillir une note globale. Nous avons obtenu 541 points, ce qui est satisfaisant par rapport aux exigences du Prix européen de la qualité (tableau 2), à savoir une approche bien 
TABleau 2

Calcul du total

\begin{tabular}{lccc}
\hline Critères & Note attribuée & Facteur & Points attribués \\
\hline - Leadership & 54 & 1 & 54 \\
- Déploiement de la stratégie et de la politique & 50 & 0,8 & 40 \\
- Gestion du personnel & 61 & 0,9 & 55 \\
- Processus & 55 & 1,4 & 77 \\
- Satisfaction des clients & 55 & 2 & 110 \\
- Satisfaction du personnel & 53 & 0,9 & 48 \\
- Gestion des documents & 1 & 0,9 & 64 \\
- Outils du management de la qualité totale & 48 & 1,4 & 67 \\
- Rôle du pouvoir institutionnel & 37 & 0,7 & 26 \\
\hline Total des points attribués & & $\mathbf{1 0}$ & $\mathbf{5 4 1}$ \\
\hline
\end{tabular}

Note: Chaque note est multipliée par le facteur approprié pour obtenir des points. Ensuite, nous avons additionné les points attribués à chaque critère pour obtenir le total des points alloués.

planifiée, documentée et sa mise en œuvre dans la plupart des fonctions visibles dans la culture de l'entreprise.

Les entreprises recherchent dans ce type d'évaluation d'un modèle de management de nouvelles opportunités d'amélioration et une meilleure conduite du processus de progrès permanent. Elles y voient aussi une nouvelle source de motivation pour ce processus. Par contre, la pression des clients, des concurrents ou de toute instance réglementaire ne semble pas constituer un argument impératif pour développer cette pratique (contrairement à la certification, par exemple). C'est donc bien dans une perspective purement managériale que s'inscrit cette démarche. Dans certains cas, elle est adaptée pour mieux tenir compte de la stratégie de l'entreprise. Le processus d'auto-évaluation est alors conçu et perçu comme l'un des outils clés de la réalisation de cette stratégie. De ce fait, les motivations qui ont conduit les entreprises de notre échantillon à introduire la démarche qualité sont représentées selon le degré d'importance (figure 3).

\subsection{Valeurs assignées aux outils de management de la qualité dans les PMI}

Les années 1980 ont exigé des entreprises d'importants efforts de productivité. Ceux-ci sont devenus une condition nécessaire de croissance et de compétitivité. Pour juger de la solidité d'une PMI, on ne peut donc plus aujourd'hui se contenter d'analyser son bilan. Il faut aussi être capable d'apprécier ses performances productives et de la situer par rapport à ses concurrentes. Il faut savoir, en d'autres termes, mesurer l'efficience avec laquelle elle gère ses ressources et prend ses décisions de production (Dietsch, 1997). Par conséquent, les outils de la qualité représentent 
Figure 3

Motivation de l'introduction d'une démarche qualité

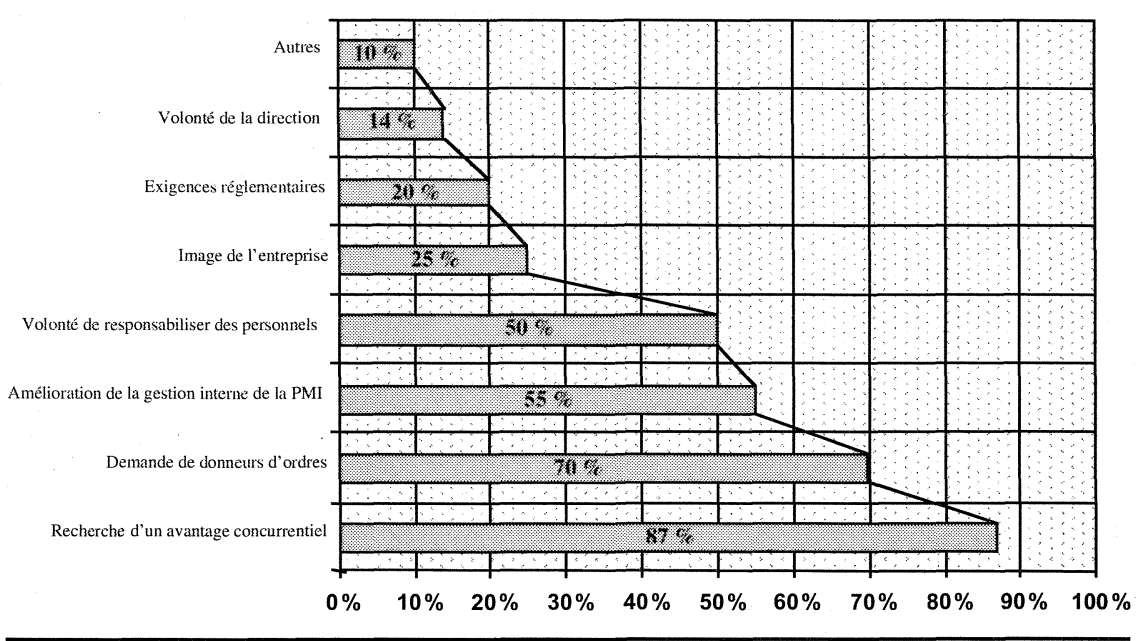

les moyens pratiques servant à résoudre les problèmes que rencontre l'entreprise tout au long de sa démarche. Le choix de ces outils sera fonction de la stratégie adoptée par l'entreprise. Ils sont utilisés dans le but de donner une information précise sur l'aptitude d'un procédé ou d'un processus et sur la conformité d'une fabrication. Ils permettent également de prévoir le résultat du processus et de prendre les dispositions nécessaires en minimisant les risques. À cet effet, des outils ont été retenus et proposés aux responsables des entreprises étudiées. La figure 4 indique pour chaque outil la répartition des réponses.

L'analyse de ces résultats montre que le développement des outils et l'implication du personnel constituent le premier objectif visé par les responsables lors de la mise en place du concept de la qualité totale. Bien que $48 \%$ des entreprises appliquent les outils de la qualité totale, il faut noter que la moitié d'entre elles seulement ont pour objectif d'atteindre le «zéro défaut ». Les entreprises concernées considèrent qu'un tel objectif permet une amélioration constante de la qualité, tandis que pour les autres, le «zéro défaut » n'aboutit qu'à fixer des standards inaccessibles, donc démotivants ${ }^{3}$. Parmi les techniques peu utilisées figurent également les techniques de déploiement de la fonction qualité (18\%). Observons que toutes ces techniques sont surtout utilisées pour le développement des produits.

Il est également remarquable que toutes les entreprises n'accordent pas une attention importante aux gains procurés par les cercles de qualité $(17 \%)$ et le suivi

3. Ministère de l'Industrie (1990), Vers la compétitivité globale, PMI 90, Paris. 


\section{FIGURE 4}

\section{Répartition des outils dans les PMI de l'échantillon}

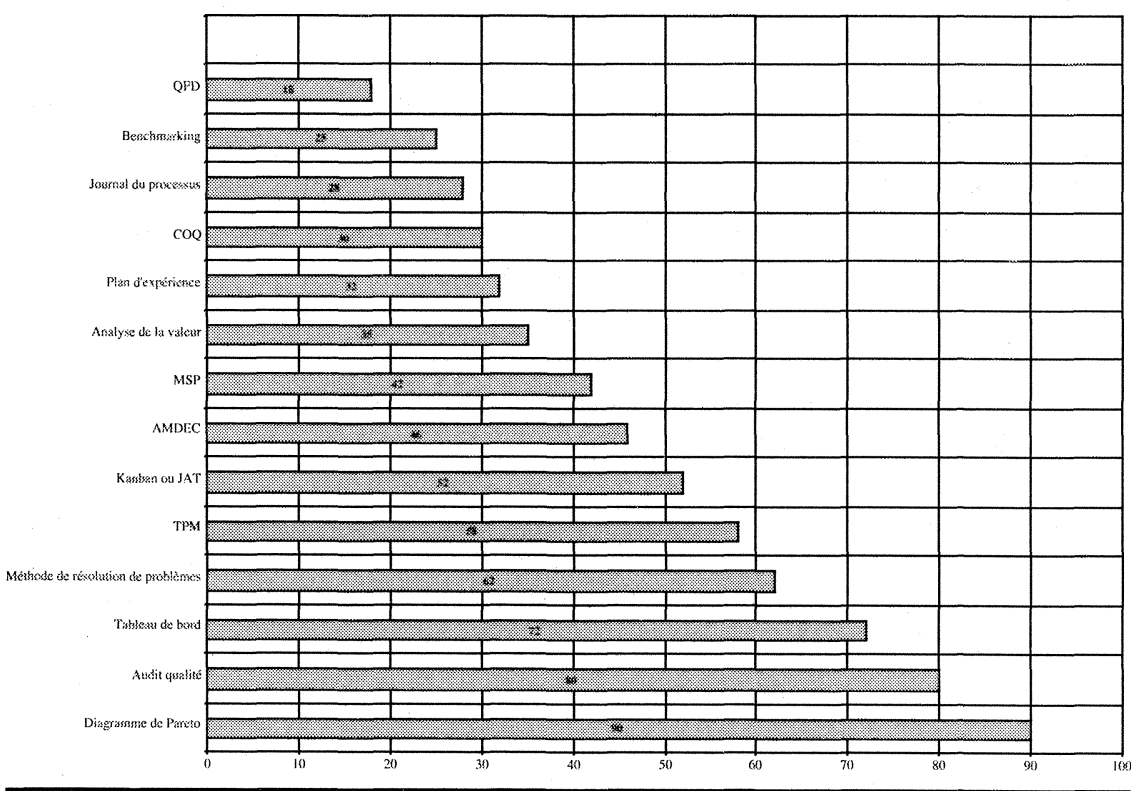

commercial $(25 \%)$. À ce niveau, $85 \%$ des entreprises se contentent de former le personnel aux méthodes d'amélioration de la qualité. La formation en interne est vitale pour tous les secteurs concernés. De plus, toutes les entreprises ont développé des indicateurs de performance et sont très ouvertes pour partager l'information à tous les niveaux sur la politique suivie. Cela permet aux employés de mieux contrôler leur travail, d'ajuster leurs efforts aux objectifs globaux de l'entreprise par l'application des outils du management de la qualité totale. C'est précisément dans cette problématique que s'inscrit l'évolution de la fonction qualité. Le rôle des responsables des PMI consiste désormais à offrir des formations adaptées à des besoins changeants, au moyen de technologies elles-mêmes en constante évolution.

Dans cette perspective, les cercles de qualité ne sont appréciés que pour leur capacité à améliorer les connaissances et la motivation des salariés en matière de qualité, ainsi qu'à faciliter les opérations de transfert de savoir-faire. Certains constats sont intéressants à mentionner. Les méthodes AMDEC (46\%), le tableau de bord qualité ( $72 \%)$, le coût d'obtention de la qualité (30\%) et l'audit qualité ( $80 \%)$, le Kanban ou juste-à-temps $(52 \%)$ et le total productif maintenance (58\%) sont bien appréciés pour leur contribution à l'obtention des performances requises.

L'analyse de la valeur qui se distingue surtout par sa capacité à permettre d'atteindre le rapport qualité / prix attendu $(35 \%)$ et la maîtrise statistique / processus 
(42\%) obtiennent leurs meilleures appréciations. L'efficience du développement des outils de la qualité totale présente le meilleur potentiel pour favoriser le développement de ce concept.

Les responsables d'entreprise n'attendent pas du fonctionnement des outils un impact direct sur l'amélioration de la compétitivité de l'entreprise, mais plutôt une augmentation de l'adhésion et de l'implication des personnes aux objectifs de l'entreprise, condition de base dans la recherche d'une plus grande compétitivité de l'entreprise. La mise en place des outils de la qualité permet à chaque salarié d'être concerné, d'être considéré, d'être reconnu. Cette prise en considération est la condition de changement de l'innovation dans l'entreprise.

\subsection{Valeurs assignées à la nature du changement par la qualité dans les PMI}

Nous avons relevé les principales attributions du concept de la qualité sur le développement de l'adhésion et de l'implication, sur l'amélioration des relations et des conditions de vie au travail. Or, la gestion a toujours consisté en un ensemble de pratiques visant à promouvoir les attitudes, les comportements et les normes qui peuvent améliorer l'efficacité de l'entreprise. Elle s'efforce de faire adhérer le personnel d'une organisation à la même idéologie. Cette dernière peut se définir comme un système organisé d'idées et de valeurs (Denfeld-Wood, 1997). De quelle manière se manifestent ces différentes valeurs dans l'organisation ? Pour obtenir ces résultats, nous nous sommes intéressé à la nature des changements intervenus au sein des PMI. Pour chacun des éléments énoncés, nous avons demandé aux responsables d'entreprise de préciser s'il y avait eu : une amélioration / aucun changement.

Dans $75 \%$ des entreprises, on note une amélioration de la motivation et de l'intérêt au travail des salariés et, dans $42 \%$ des cas, une augmentation du sentiment d'appartenance à l'entreprise. Cette structuration dans les entreprises se traduit par une réduction des écarts hiérarchiques, un élargissement des responsabilités des opérateurs et un travail plus collectif.

En ce qui concerne la responsabilisation du personnel aux problèmes de qualité, la majorité des entreprises considère qu'elle est possible. Les méthodes de responsabilisation évoquées sont l'information (75\%), la formation (83\%) et l'intéressement « prime» $(42 \%)$.

Informer le personnel constitue donc l'opinion la plus fréquemment exprimée, comme facteur de responsabilisation en matière de qualité. Pour ce qui est des moyens de développer la qualité, la majorité des PMI affirme être d'accord avec l'opinion selon laquelle toute amélioration de la qualité passe par la motivation du personnel et son implication. À cet effet, les changements les plus souvent constatés par les entreprises sont significatifs et importants (figure 5). 
FIGURE 5

Attribution des réponses apportées par les PMI sur la nature du changement

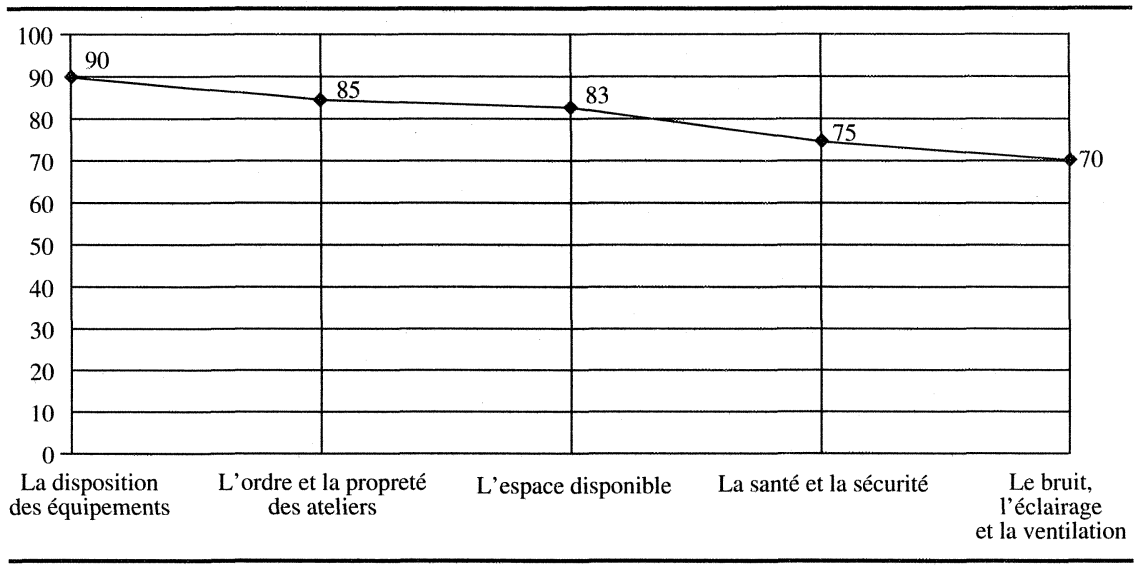

Nous pouvons noter qu'une majorité d'entreprises a enregistré un changement dans les programmes de formation $(83 \%)$, de communication $(60 \%)$ et dans le fonctionnement des autres structures participatives $(62 \%)$. Les dépenses de formation $^{4}$ par salarié augmentent quelle que soit la taille des entreprises. Il semble que la majorité des PMI de l'échantillon fait des efforts d'information et d'analyse sur l'environnement de l'entreprise et évalue les conséquences financières de ses décisions. L'image de la PMI sous-gérée est probablement dépassée. La formation du personnel revêt une importance considérable. La démarche qualité met en évidence les buts poursuivis.

\section{Indicateurs apparents de l'étude}

Les résultats de l'analyse descriptive, la perception du concept de la qualité des dirigeants en PMI et les pratiques des processus de gestion de la qualité sont successivement présentés.

\subsection{Analyse globale des résultats}

Le résultat de cette étude fait ressortir que la majorité des PMI affirme avoir la maîtrise de la gestion de la qualité. Ces entreprises font appel à la panoplie des outils et méthodes (48\%). Par ailleurs, nombre d'entre elles déclarent s’être engagées dans

4. L'enquête annuelle de l'AFGEFOS-PME 1997 révèle une augmentation des coûts de formation par salarié, quelle que soit la taille des entreprises. 
la voie du management participatif : information et formation des opérateurs, mise en place de cellules de réflexion, de groupes de résolution des problèmes $(62 \%)$. Mais très peu d'entreprises parviennent à chiffrer les gains que l'on peut espérer de la démarche d'un management participatif (17\%) et s'appuient donc sur des éléments qualitatifs, comme l'amélioration de l'image de l'entreprise.

Cette propension des PMI à se doter d'un service qualité peut être interprétée comme un besoin et une capacité de mieux contrôler la gestion de l'entreprise face à la complexité croissante d'un système de production. L'existence d'une fonction qualité favorise également la coordination entre les différents services. Pour que l'autocontrôle du fournisseur et les rapports d'évaluation soient crédibles, la majorité des entreprises font confiance aux documents écrits beaucoup plus qu'elles ne l'ont fait traditionnellement. Avec le passage de la coordination marchande à la coordination industrielle, la communication écrite change avec la rédaction du manuel qualité dans toutes les entreprises. Le manuel qualité devient, en somme, un instrument majeur d'introduction de l'esprit de discipline et de méthode, indispensable à la coordination industrielle.

En ce qui concerne les procédures de recherche d'amélioration de la qualité qui s'inscrivent, non plus dans un univers subi, mais dans un univers volontariste, on observe que $78 \%$ des entreprises ont clairement défini les objectifs et les politiques qualité. Elles affirment se réunir au moins une fois par an pour définir les objectifs opérationnels sur la qualité. Des contacts structurés existent entre les différentes fonctions de l'entreprise et croissent avec la taille de celle-ci. Concernant les relations avec l'extérieur, $70 \%$ des entreprises sont en contact avec des responsables qualité appartenant à d'autres entreprises, $37 \%$ avec des institutions et d'autres associations. La perception de l'action publique par les entreprises est diverse mais traduit, dans la plupart des cas, une connaissance partielle restrictive, voire erronée, de l'action publique. Cette situation est sans doute liée aux difficultés d'accès à l'information dans la sphère de proximité de l'entreprise, à la dispersion et à la multiplicité des messages reçus.

Les souhaits des entreprises à l'égard de l'action des pouvoirs publics s'organisent autour de plusieurs pôles :

- un accès plus direct à l'information sur la qualité et sur les actions publiques ;

- un renforcement de la présence locale de leurs interlocuteurs privilégiés et un suivi personnalisé ;

- un allégement des procédures administratives ;

- une reconnaissance des certifications au niveau européen.

Dans ce contexte, le Mouvement français pour la qualité et l'AFNOR sont perçus comme pouvant constituer un lieu privilégié d'information et de promotion 
à l'échelon national et international. L'analyse des performances de ces entreprises est également sans ambiguïté, tant la valeur ajoutée par emploi que la rentabilité des entreprises évoluent de façon croissante. Un autre constat majeur est l'importance du phénomène européen. L'Europe est un pari gagnant pour les PMI qui en font de plus en plus leur marché. Dans le cadre de l'initiative communautaire en faveur des PME-PMI, la Commission européenne a prévu de soutenir le développement de réseaux d'entreprises mettant notamment en relation les entreprises des régions les plus avancées avec celles des régions moins développées. Elle apporte également des cofinancements à des actions visant à encourager le regroupement des sous-traitantes en réseaux de production ou de distribution (dans le domaine d'achat, par exemple). Les sous-traitances sont considérées, d'une part, comme un facteur clé de la compétitivité des entreprises et, d'autre part, elles constituent un des éléments majeurs du développement économique et de l'emploi en Europe (Pras et Boutin, 1995).

\subsection{Commentaire sur les résultats}

Les résultats de cette étude sont importants à un double niveau :

- bien que le bilan économique et social du concept de la qualité se révèle positif dans une majorité d'entreprises, les démarches apparaissent comme une obligation imposée par les donneurs d'ordres, dans le cadre de la certification ISO 9000 ; elles ne peuvent répondre qu'à des objectifs limités et doivent donc être intégrées dans une politique plus globale de mobilisation des hommes;

- le concept de la qualité totale, pour fonctionner et apporter le maximum d'efficacité, a besoin d'un réel suivi et d'une évolution de l'organisation, en particulier, du système de gestion des ressources humaines.

D'autres hypothèses ont été mises à l'épreuve. Compte tenu des débats actuels relatifs à la stratégie de qualité, deux d'entre elles sont particulièrement intéressantes à commenter.

La première prévoit que les entreprises qui ont mis en place un système de contrôle de la qualité totale atteindront mieux leurs objectifs que les entreprises où un tel système est absent. En effet, les entreprises disposant seulement d'un système d'assurance qualité sont celles qui réalisent le mieux leurs objectifs. Ce résultat peut s'expliquer par le fait qu'un concept de la qualité totale ne suffit pas à garantir les résultats et qu'il existe un écart significatif entre les aspirations liées à un tel système et les actions menées concrètement au niveau des différentes fonctions pour que les aspirations soient ancrées dans la qualité.

Selon la seconde hypothèse importante, l'efficacité des instruments mis au service de la qualité est corrélée à l'engagement des dirigeants. Cette hypothèse 
est en partie vérifiée, dans la mesure où il se révèle bien qu'un fort appui de la direction permet de mieux atteindre les objectifs que lorsqu'il n'est que moyen (Liouville, 1992).

Nous estimons que les PMI doivent avant tout aider les personnels à modifier leur comportement. Il ne sert à rien de vouloir se protéger de la concurrence de sociétés plus performantes par des réglementations. Il est préférable de savoir, et, avant tout, de vouloir s'adapter pour survivre. C'est propre aux êtres vivants de s'adapter aux changements. S'ils ne le font pas, ils disparaissent. Il n'appartient qu'à nous de forger notre destin, en s'appuyant mieux sur les règles qui régissent les hommes ${ }^{5}$.

C'est pourquoi les PMI doivent favoriser l'amélioration de la qualité de tous les hommes de l'entreprise : les employés qui sont au sein de la firme doivent savoir informer, responsabiliser et considérer chacun des membres du personnel de l'entreprise. Il faut satisfaire les trois besoins fondamentaux psychologiques des hommes : besoin de savoir, besoin de pouvoir, besoin de considération. La satisfaction de ces trois besoins est presque aussi indispensable pour chacun d'entre nous que les besoins physiologiques. Celui qui n'est pas satisfait déclenche les révoltes, voire les révolutions, hier comme aujourd'hui. C'est ce que Serieyx (1991) appelle le zéro mépris. Cette considération doit s'appliquer non seulement au personnel de l'entreprise, mais aussi aux clients, aux fournisseurs. La considération, c'est la reconnaissance de l'existence des autres, c'est s'intéresser à eux, c'est se préoccuper de leurs problèmes, c'est les féliciter quand ils le méritent. Le manque de considération fait autant de tort à un responsable placé en amont de la hiérarchie qu'à un opérateur oublié dans son atelier (De Fournas, 1997).

Ces trois besoins psychologiques relègue au deuxième plan le seul besoin matériel dont les entreprises, les syndicats et les gouvernements se sont préoccupés jusqu'à présent : le besoin d'avoir, qu'on a tenté de satisfaire par des augmentations de salaires. La satisfaction seule de ce besoin ne résout rien et compromet tout, car il ne sera jamais assouvi.

Les enquêtes sociologiques révèlent aussi qu'une source majeure de satisfaction réside dans le respect, la confiance accordée à chacun pour agir sur la situation du travail, améliorer l'efficacité et intervenir utilement au service des objectifs de l'entreprise. Il est temps d'interroger le personnel sur le climat interne, mais aussi sur les gisements de qualité, les attentes perçues de la clientèle, les types de services qui pourraient être rendus, la perception des points forts et des points faibles de l'entreprise au regard de son marché et de la concurrence. C'est avec ce type de connaissance enrichie et partagée que l'on rendra chacun acteur

5. Cf. Qualité en mouvement, $\mathrm{n}^{0} 5,1992$. 
du mouvement. Une telle démarche de connaissance et de mesure s'avère un gisement précieux d'informations pour un meilleur développement des ressources humaines, mais aussi un puissant levier de mobilisation pour éliminer les dysfonctionnements (Le Youdec, 1996).

En outre, les PMI doivent chercher à rapprocher de plus en plus le savoirfaire de l'endroit où l'on crée la richesse, dans les ateliers comme dans les bureaux. Ceci implique une autre manière de fonctionner, avec en particulier une simplification de l'organisation qui est non seulement un objectif, mais aussi la résultante d'une plus grande compétence et d'une plus grande autonomie de chaque personne sur le terrain. Toutes les entreprises doivent comprendre qu'il faut développer une autre façon d'exercer le pouvoir, de faire accepter les changements au niveau de la hiérarchie intermédiaire qui doit évidemment abandonner certaines attributions de son rôle traditionnel.

Face à cette évolution indispensable, la responsabilité la plus importante pour le management est bien d'indiquer le sens dans lequel va l'entreprise et d'exprimer la volonté de réussir qui doit animer chacun de ses acteurs. Donc, il n'y a pas de valeurs spécifiquement rattachées à la qualité, mais plutôt à un système de management dans sa totalité. Nous en distinguons trois, qui sont essentielles : la transparence, c'est-à-dire une libre circulation de l'information, la confiance, sans laquelle les gens ne peuvent prendre des responsabilités, ce qui implique le droit à l'erreur, et, enfin, le respect des personnes, quelle que soit leur position hiérarchique. Il faut en fait passer du management de la qualité à la qualité de management.

\section{Conclusion}

Les résultats obtenus à partir de la vérification empirique du modèle confirment que le concept de qualité totale semble bien répandu et que ses enjeux sont bien perçus, autant par les entreprises du secteur industriel que par les autres PMI. Ainsi, $83 \%$ des entreprises répondantes déclarent avoir une politique de qualité totale. Elles considèrent qu'adopter ses principes permet d'assurer la qualité de la prestation et, par conséquent, de satisfaire totalement le client tout en luttant contre la concurrence. Donc, la satisfaction du client est prise en considération par le service qualité. Enfin, les succès réalisés grâce à la qualité totale sont en général plus visibles dans une PMI et peuvent servir à promouvoir ou à appuyer la philosophie de celleci. Mais, à travers cette analyse, nous pouvons constater que si les PMI se préoccupent et s'intéressent aux outils de la qualité, très peu d'entre elles recourent à des démarches structurées, capables de fournir un outil de gestion à l'entreprise (suivi commercial, calcul du coût entraîné par la qualité et développement du management participatif). Cependant, cette enquête permet de prévoir que cette situation ira en s'améliorant compte tenu de plusieurs facteurs : la prise de conscience d'ores et déjà effectuée, la jeunesse de la plupart de ces démarches, l'évolution et la vulgarisation 
de ce concept, la pression concurrentielle qui obligera les entreprises à s'y plier, le soutien apporté à ce concept par des politiques de qualité totale désormais pratiquement incontournables.

De ce fait, le processus de qualité se développe dans les petites entreprises de la même façon que dans les grandes sociétés. Les PMI sont des entreprises caractérisées par quelques principes spécifiques : leurs buts sont ceux du dirigeant, directement issus de sa perception de l'environnement. Elles ont une structure souple et changeante et recherchent dans la qualité une image et une légitimité qui exerceront un attrait sur des clients potentiels. Il faut noter que les PMI peuvent aussi, et indépendamment d'un donneur d'ordres, être engagées dans des processus de qualités stratégiques parce que le dirigeant perçoit un besoin ou qu'il envisage une stratégie d'expansion centrée sur une image de qualité, voire de qualité totale. Les répercussions d'une telle démarche, ainsi que son intégration dans les PMI doivent être l'affaire de tous pour être réussies (Guilhon et Weill, 1995). Toutefois, il parait possible d'émettre quelques conclusions qui ressortent de l'application de la démarche qualité sur les processus clés de l'entreprise :

- la nécessité de trouver de nouveaux moyens pour améliorer les performances globales des entreprises est bien établie et universellement reconnue. Transformer cette nécessité en un programme d'amélioration bien planifié et fructueux, toutefois, est un défi que bien peu d'organisations parviennent à relever avec succès, et la plupart ne tentent même pas de le faire. Le défi consiste à trouver la meilleure façon de planifier et de mettre en œuvre un programme d'amélioration continue ;

- pour commencer rapidement et pour conserver l'élan d'une organisation, il est essentiel que le programme ne soit pas élaboré sous une forme particulière. Ceci doit être compréhensible par tous : il sera diffusé à tous les niveaux de l'organisation et présentera des objectifs clairs dès le départ. Il lui faut également être crédible, constant et susceptible d'être connu. C'est tout un défi !

De ce fait, la combinaison d'un processus de qualité totale bien défini et des critères associés à d'autres méthodologies permet d'améliorer la coopération entre les différents services. Les diverses entreprises peuvent aussi mettre en commun leurs meilleures idées et méthodes de mise en œuvre de qualité et de satisfaction de la clientèle.

\section{Bibliographie}

ANSARI, M. (1999), «Motivation concept within educative organization », Colloque international «TQM for Higher Education Institutions », Università di Verona, Italie, 30-31 août. 
ANSARI, M. (1998), «La certification ISO 9000 dans les entreprises : entre nécessité et contraintes », Colloque international «Les décisions sous contraintes », Université de Caen, 26-27 novembre.

AUREGAN, P. (1996), L'influence des attributs temporels sur les perceptions et les comportements du dirigeant d'entreprise, Thèse de doctorat, Université de Caen, dans B. Saporta, Stratégie et PME, VII Journée nationale des IAE, Poitiers, 18-20 novembre 1987, vol. 2, p. 1059.

BALlot, E. (1996), «Du nouveau en économie de E. Deming », Revue française de gestion, $\mathrm{n}^{\circ} 1$, p. $58-60$.

Belley, J.G. et A. Billette (1995), «Les PME et les risques de la sous-traitance industrielle à l'heure de l'assurance qualité », Revue internationale PME, vol. 8, $\mathrm{n}^{\circ} 2$, p. 49-77.

BONNET, E. (1994), Analyse sociologique des actions qualité dans l'entreprise industrielle. Des visions indigènes en représentation, Thèse de doctorat, Paris X-Nanterre.

CAlori, R., P. Very et J.L. ArRegle (1997), «Les PMI face à la planification stratégique », Revue française de gestion, $\mathrm{n}^{\circ}$ 112, janvier-février, p. 11-23.

Coue, D. et A. REMOuE (1996), « S'adapter à l'européanisation des marchés », L'Usine nouvelle, $\mathrm{n}^{\circ} 2572,28$ novembre, p. 52-58.

CROSNIER, P. (1990), «PMI tricolore : $20 \%$ des exportations industrielles », Moci, $\mathrm{n}{ }^{\circ} 952$, 24 septembre, p. 106-111.

CrozIER, M. (1985), Les nouveaux modes d'organisation, Paris, Éditions Institut de l'Entreprise, p. 28.

De FouRnAS, F.X. (1997), «Vous avez dit qualité », Revue de banque, no 580, p. 44-46.

DEMING, W.E. (1991), Hors de la crise, Paris, Economica.

DENFELD-WoOD, J. (1997), «La structure n'explique pas tout, l'Art du management », Les Echos, vendredi 28 février et $1^{\mathrm{er}}$ mars.

DIETSCH, M. (1997), «L'efficience : un nouvel outil de mesure des performances », Revue banque, $\mathrm{n}^{\circ} 586$, novembre, p. 52-55.

Douchy, J.M. (1990), Vers le zéro défaut dans l'entreprise, Paris, Dunod Entreprise.

EFQM (1996), Cahiers d'auto-évaluation, Rapport de la Fondation européenne pour le management de la qualité, Bruxelles.

ESPOSITO, E. et M. RAFFA (1992), «Qualité et sous-traitance dans l'industrie italienne : quelques résultats d'une étude empirique », Revue internationale PME, vol. 5, $\mathrm{n}^{\mathrm{o}} 2$, p. 57-82.

FORISSIER, N. (1995), Mobiliser nos entreprises sur le marché intérieur européen : rapport et proposition, Paris, La Documentation française.

FOURCANS, A. (1993), L'entreprise et l'Europe, clés pour le décideurs, Paris, InterÉditions, p. 191-204.

GAGNEPAIN, F. (1997), «Brisa sauvé par la qualité totale », Qualité en mouvement, $\mathrm{n}^{\circ} 31$, p. $39-42$. 
GÉLINIER, O. (1993), Stratégie de l'entreprise et motivations de l'homme, Paris, Les Éditions d'Organisation, p. 165.

Gogue, J.M. (1987), Qualité et productivité, même combat, Paris, Éditions du Moniteur.

Gomez, P.Y. (1996), «Normalisation et gestion de la firme: une approche conventionnaliste », Revue d'économie industrielle, $\mathrm{n}^{\mathrm{o}} 75,1^{\mathrm{er}}$ trimestre, p. 113-131.

GuILHON, A. et M. WeILl (1995), «Démarche qualité : de la stratégie d'adaptation aux processus stratégiques de changements dans les $\mathrm{PME} »$, Résultat d'un test empirique, Communication de la $51^{\mathrm{e}}$ conférence AIMS, Lille, mai.

IGALENT, J. (1992), «Image de la gestion des ressources humaines dans le discours de la qualité », Colloque de l'AGRH, Lille, novembre, p. 143-161.

JAVEAU, C. (1994), L'enquête par questionnaire, Bruxelles, Éditions de l'Université de Bruxelles, $138 \mathrm{p}$.

JoCOU, P. et F. LuCAS (1992), Au cœur du changement, Paris, Dunod.

JUSLIN DE NORAY, B. (1993), «La certification ISO 9000 », Qualité en mouvement, $\mathrm{n}^{\circ} 13$, p. 24-40.

KeladA, J. (1994), «Pas de reengineering sans qualité totale», L'Expansion et Management Review, $\mathrm{n}^{\circ} 73$, été, p. 60-65.

Krebs, A. (1997), «Qualité et union européenne», Qualité en mouvement, no 29 , p. $36-40$.

LEFEBVRE, E. et M. BourgaUlt (1996), «Performance à l'exportation technologique dans les PME manufacturières », Revue d'économie industrielle, $\mathrm{n}^{\circ} 77$.

LE Youdec, M.H. (1996), «Qualité et motivation : quels facteurs clés, quels points d'impact », Annales des mines, avril-mai, p. 86-92.

Liouville, J. (1992), «Gestion de la qualité dans la phase de conception des produits en Allemagne », Analyse de la SEDEIS, n 90 , p. 33-37.

MARTIN, Y. (1992), «La sous-traitance au Québec », Centre d'études en administration internationale (CETAI), Montréal, École des Hautes Études commerciales, p. 195-198.

MCMillan, J. (1996), «Une politique européenne pour la promotion de la qualité », Annales des mines, avril-mai, p. 23-27.

Mispelblom, F. (1995a), Au-delà de la qualité, démarche qualité, conditions de travail et politique du bonheur, Paris, Éditions Syros.

MisPelblom, F. (1995b), «La qualité : une politique sociale des biens et des services », Annales des mines, avril-mai, p. 112-119.

MORET, J. (1991), Objectif qualité, le témoignage d'un chef d'entreprise, Paris, Éditions du Mouvement français pour la qualité.

NeUville, D. (1996), «La qualité en question», Revue française de gestion, $\mathrm{n}^{\circ} 108$, mars-avril-mai, p. 37-48.

NGUYEN, N. (1991), «Un modèle explicatif de l'évaluation de la qualité d'un service : une étude empirique », Recherche et application en marketing, vol. 6, $\mathrm{n}^{\circ} 2$, p. 83-98.

PERIGORD, M. (1987), Réussir la qualité totale, Paris, Les Éditions d'Organisation. 
PEYRAUT, Y. (1988), Gestion rationnelle de la qualité, Paris, Éditions Entreprise Moderne.

Pras, B. et A. Boutin (1995), Les Euro-PMI, Paris, Economica, 224 p.

PRICE, M. et E. CHEN (1994), «Conduire un "start-up" vers l'âge de raison », L'Expansion et Management Review, $\mathrm{n}^{\circ}$ 73, été, p. 66-75.

RAVIX, J.T. et P.M. RoMANI (1996), «Certification et forme de coordination dans l'organisation de la production industrielle », Revue d'économie industrielle, $\mathrm{n}^{\circ} 75$, $1^{\text {er }}$ trimestre, p. 275-290.

ROUSSEAU, A. et G. WARNOTTE (1994), «La responsabilisation des cadres face à la qualité totale : un construit social ? », Annales des mines, décembre, p. 31-40.

SAVAL, H. et V. ZARDET (1989), Maîtriser les coûts et les performances cachés, Paris, Economica.

SERIEYX, H. (1991), Le zéro mépris, Paris, InterÉditions, 233 p.

WARNOTTE, G. (1992), «La qualité totale : fondements, principes d'actions et enjeux », Humanisme et Entreprise, Paris, p. 4-18.

ZiANE, E. (1993), Maîtrise de la qualité totale, Paris, Hermès.

ZOBRIST, F. (1996), «Une démarche qualité dans une PMI Picardie », Réalités industrielles, avril-mai, p. 83-85. 